\title{
Infarto agudo do miocárdio: Do diagnóstico à intervenção
}

\author{
Acute myocardial infarction: From diagnosis to intervention \\ Infarto agudo de miocardio: Del diagnóstico a la intervención
}

Recebido: 02/02/2022 | Revisado: 07/02/2022 | Aceito: 13/02/2022 | Publicado: 20/02/2022

Murilo Santos Bett
ORCID: https://orcid.org/0000-0001-6102-1137
Universidade do Planalto Catarinense, Brasil
E-mail: caiambora @ gmail.com
Julia Melin Zardo
ORCID: https://orcid.org/0000-0002-7876-3417
Universidade do Planalto Catarinense, Brasil
E-mail: juliamelim @ uniplaclages.edu.br
Jessica Lie Utiamada
ORCID: https://orcid.org/0000-0003-3981-4311
Universidade do Planalto Catarinense, Brasil
E-mail: jeeeutiamada @ gmail.com
Juliana Lessmann Reckziegel
ORCID: https://orcid.org/0000-0003-4747-4038
Universidade do Planalto Catarinense, Brasil
E-mail: julianalessmann @gmail.com
Vanessa Valgas dos Santos
ORCID: https://orcid.org/0000-0002-9554-3045
Universidade do Planalto Catarinense, Brasil
E-mail: vanessavalgas@ uniplaclages.edu.br

\section{Resumo}

O Infarto Agudo do Miocárdio constitui uma das principais causas de óbito tanto no Brasil quanto no mundo. Um dos grandes problemas relacionados à morbimortalidade da doença é o tempo entre o inicio dos sintomas e inicio do tratamento. Assim, o objetivo deste artigo de revisão narrativa, foi ressaltar a importância da atuação precoce e das intervenções farmacológicas e não farmacológicas realizadas nos pacientes infartados. Para isto, foi realizada uma busca nas bases de dados Pubmed, Biblioteca Virtual em Saúde e Sciencedirect através das palavras-chave "Myocardial Infarction, Acute Myocardial Infarction, ST Elevation Myocardial Infarction, Myocardial Reperfusion, Reperfusion Injury" e Infarto do Miocárdio, Infarto Agudo do Miocárdio, Infarto do Miocárdio com Elevação de ST, Reperfusão do Miocárdio, Lesão de Reperfusão. Os principais achados ressaltaram a importância da eletrocardiografia no diagnostico associada a marcadores bioquímicos e de imagem, as intervenções necessárias para a restauração da irrigação e os medicamentos como analgésicos, anti-agregantes e terapia fibrinolíticas. Assim, reforça-se a necessidade de busca médica aos primeiros sinais e sintomas, para que a identificação e as intervenções necessárias sejam realizadas na tentativa de manutenção da qualidade de vida.

Palavras-chave: Infarto agudo do miocárdio; Infarto agudo do miocárdio com elevação do segmento ST; Reperfusão do miocárdio; Lesão de reperfusão.

\begin{abstract}
Acute Myocardial Infarction is one of the main causes of death in Brazil and in the world. One of the major problems related to the morbidity and mortality of the disease is the time between the onset of symptoms and the beginning of treatment. Thus, the purpose of this narrative review article was to reinforce the importance of early action and pharmacological and non-pharmacological interventions performed in infarcted patients. For this, the search was carried out in the Pubmed, Virtual Health Library, and Sciencedirect databases using the keywords Myocardial Infarction, Acute Myocardial Infarction, ST-Elevation Myocardial Infarction, Myocardial Reperfusion, Reperfusion Injury, and Infarto do Miocardio, Infarto Agudo do Miocardio, ST-Elevation Myocardial Infarction, Myocardial Reperfusion, Reperfusion Injury. Accumulating data show electrocardiography importance at the diagnostic associated with biochemical parameters, and image analysis. The data also reveal the necessity of therapies for irrigation restoration based on anti-aggregating and fibrinolytic. Thus, it reinforces the need to search for medical assistance at the first signs and symptoms, for posterior identification and intervention out in an attempt to maintain the quality of life.
\end{abstract}

Keywords: Myocardial infarction; Acute myocardial infarction; ST-elevation myocardial infarction; Myocardial reperfusion; Reperfusion injury. 


\begin{abstract}
Resumen
El Infarto Agudo de Miocardio es una de las principales causas de muerte en Brasil y en el mundo. Y uno de los grandes problemas relacionados con la morbimortalidad de la enfermedad es el tiempo transcurrido entre el inicio de los síntomas y el inicio del tratamiento. Así, el objetivo de este artículo de revisión narrativa fue enfatizar la importancia de la actuación precoz y de las intervenciones farmacológicas y no farmacológicas realizadas en pacientes infartados. Para ello se realizó una búsqueda en las bases de datos Pubmed, Virtual Health Library y Sciencedirect utilizando las palabras clave "Myocardial Infarction, Acute Myocardial Infarction, ST Elevation Myocardial Infarction, Myocardial Reperfusion, Reperfusion Injury" y Myocardial Infarction, Acute Infarction of Myocardium, ST Elevación del infarto de miocardio, reperfusión miocárdica, lesión por reperfusión. Los principales hallazgos destacaron la importancia del electrocardiograma en el diagnóstico asociado a marcadores bioquímicos y de imagen, las intervenciones necesarias para restaurar la irrigación y medicamentos como analgésicos, antiplaquetarios y terapia fibrinolítica. Así, se refuerza la necesidad de buscar atención médica ante los primeros signos y síntomas, para que se realicen las identificaciones e intervenciones necesarias para mantener la calidad de vida.

Palabras clave: Infarto de miocardio; Infarto agudo de miocardio; Infarto del miocardio con elevación ST; Reperfusión miocárdica; Lesión por reperfusión.
\end{abstract}

\title{
1. Introdução
}

As doenças cardiovasculares (DCV) estão entre as principais causas de morbimortalidade, representando $31 \%$ das mortes globais. Estas doenças levam a complicações, incapacidade significativa e produtividade reduzida, sendo considerada uma patologia onerosa para o sistema de saúde com elevados custos à sociedade (Kaptoge et al., 2019).

De acordo com dados recentes do relatório da Organização Mundial da Saúde (OMS), 17,9 milhões de pessoas morrem anualmente de DCV, e prevê-se que até 2030 os óbitos chegarão a 25 milhões por ano, tornando-se a principal causa de morte no mundo ("WHO | Cardiovascular Diseases (CVDs)," 2019).

No Brasil, dentre as doenças cardiovasculares, o IAM é responsável pela primeira causa de morte no Brasil (Somuncu et al., 2019).

Muitas hipóteses surgiram sobre a origem da atual epidemia de DCV, mas entre as explicações, a mais aceita é atribuída à industrialização iniciada em 1700 (Mann et al., 2014). A adoção de uma dieta rica em carboidratos, gorduras saturadas, comidas processadas, o aumento no tabagismo e a redução da atividade física, levaram a um aumento de fatores de risco modificáveis, como a hipertensão, hipercolesterolemia e diabetes mellitus. Estes fatores de risco continuaram nos séculos XX e XXI, resultando nos valores crescentes da doença nos dias atuais (Dalen et al., 2014).

Em 2016 estimou-se que aproximadamente a cada 42 segundos, um americano sofreu um infarto do miocárdio (Mozaffarian et al., 2016). Estas mortes ocorrem nas primeiras horas da manifestação da doença, sendo de 40 a $65 \%$ na primeira hora e, aproximadamente, 80\% nas primeiras 24 horas (Abreu, 2019; Antman, 2008). Mas estes achados tiveram seu início graças à hipótese revolucionária de Eugene Braunwald, "tempo é músculo" propondo que o IAM é um processo dinâmico e seu resultado é determinado pelo tamanho do infarto e pelo tempo para o tratamento (Braunwald, 1998).

Braunwald e colaboradores propuseram que do ponto de vista clínico, era fundamental a descoberta da gravidade e da extensão da lesão isquêmica do miocárdio resultante da oclusão coronariana, porém os autores afirmavam que as mesmas poderiam ser radicalmente alteradas não apenas pelo pré-tratamento, mas também por uma intervenção apropriada até 3 horas após a oclusão coronariana (Kjekshus, 1974).

Esta teoria foi seguida do termo "hora de ouro" atribuída a R. Adams Cowley, no seu artigo de 1975 onde declarou que 'a primeira hora após a lesão determina as chances de sobrevivência'(Cowley, 1975). Isto porque os investigadores compreenderam que a recuperação do miocárdio no momento da oclusão coronariana não acontece de maneira linear e sim, curvilínea, com a quantidade máxima de recuperação nas primeiras horas após o início do infarto, com forte redução na quantidade de resgate a partir de cada hora que passa.

A seguir, em 1977 Reimer e colaboradores ressaltaram novamente a importância do tempo no tratamento do IAM.

Estes pesquisadores revelaram que IAM evolui gradualmente com uma "onda" do endocárdio em direção ao epicárdio. Desta 
forma, o tamanho final do infarto estaria relacionado não somente ao tamanho da região sujeita à isquemia, ou seja, ao miocárdio em risco, mas também com o tempo desde o início da oclusão à restauração da perfusão no miocárdio isquêmico (Reimer et al., 1977).

Os conceitos citados acima foram essenciais para criação de uma janela temporal de atendimento onde atualmente o tempo recomendado para o tratamento é 90 minutos e a "hora de ouro" ideal de até 60 minutos (Ibanez et al., 2018). Assim, o atendimento precoce aumenta as chances de sobrevida, sendo então essenciais para redução da mortalidade dos pacientes infartados.

Desta maneira, devido à gravidade do IAM, dos números elevados de casos a cada ano e da necessidade de intervenção precoce, o objetivo desta revisão narrativa de literatura é descrever sobre o diagnóstico e os tratamentos para o IAM, reforçando a importância da intervenção e do conhecimento medicamentoso para redução dos efeitos deletérios do IAM.

\section{Metodologia}

De acordo com Ribeiro (2014), a revisão narrativa apresenta uma preocupação primária em ofertar "sínteses narrativas", que permitem reunir informações de diferentes obras, mostrando para o leitor de forma clara os resultados sem a necessidade de descrever os critérios de coleta e maneira de seleção das obras incluída. Assim, na escrita desta revisão narrativa de literatura, foi realizada uma pesquisa nas bases de dados Pubmed, Biblioteca Virtual em Saúde e Sciencedirect, onde foram incluídos artigos e guidelines dentro de um recorte temporal dos últimos anos (2000-2019), com exceção dos artigos clássicos que auxiliaram na construção das teorias para a "hora de ouro" na cardiologia e nas mudanças metodológicas de tratamento $(1950,1975,1977,1992,1998)$.

Foi realizado um levantamento dos materiais referentes através da utilização das palavras-chave em inglês para sites estrangeiros e em português para sites nacionais. As palavras para busca foram respectivamente "Myocardial Infarction, Acute Myocardial Infarction, ST Elevation Myocardial Infarction, Myocardial Reperfusion, Reperfusion Injury" e Infarto do Miocárdio, Infarto Agudo do Miocárdio, Infarto do Miocárdio com Elevação de ST, Reperfusão do Miocárdio, Lesão de Reperfusão.

Os artigos incluídos foram selecionados entre os autores que concordaram com a utilização dos mesmos para a escrita deste material, sendo selecionados 47 artigos científicos.

\section{Resultados e Discussão}

\section{Eletrocardiografia na Síndrome Coronariana Aguda}

O diagnóstico de infarto do miocárdio pode ser realizado por vários métodos, mas o eletrocardiograma (ECG) é considerado um exame complementar para avaliação cardiológica rápida, de fácil realização e de baixo custo. Apesar de sua baixa sensibilidade nas síndromes coronarianas agudas, o eletrocardiograma continua sendo um exame muito utilizado capaz de fornecer dados diagnósticos, evolutivos e prognósticos das doenças cardiovasculares (Wang et al., 2017).

O ECG é uma demonstração gráfica da atividade elétrica do coração pelo tempo, que é mensurado através da colocação de eletrodos em pontos específicos (Dotta et al., 2018).

O coração possui dois tipos celulares específicos. As células musculares chamadas cardiomiócitos e as células marcapasso, responsáveis pela geração e condução dos estímulos elétricos através das diferentes câmaras cardíacas superiores (átrios) e inferiores (ventrículos), tendo em vista que estas câmaras são isoladas eletricamente entre si (Mann et al., 2014).

As células marca-passo são responsáveis pela despolarização espontânea que resultará na contração muscular cardíaca. Em condições normais, o potencial elétrico é gerado inicialmente por células fusiformes localizadas entre a veia cava superior e o átrio direito, na região denominada de Nodo Sinoatrial (NSA) (Mahmooda et al., 2014). 
O estímulo gerado no NSA flui de maneira unidirecional para a região do nodo atrioventricular (NAV), localizado anatomicamente na base do átrio direito. O NAV também diminui a atividade elétrica, dando tempo aos átrios para serem preenchidos e então, o estímulo elétrico será conduzido para região ventricular. Nos ventrículos, o impulso elétrico será conduzido pelo feixe de His e Fibras de Purkinje (Vigmond \& Stuyvers, 2016).

Sendo assim, durante o registro de ECG, observa-se a soma de todos os potenciais elétricos gerados por unidade de tempo. Cada deflexão ou onda do ECG representa despolarização e repolarização das partes específicas do coração. Como a despolarização ocorre antes da contração mecânica, as ondas de despolarização estão associadas à contração dos átrios e ventrículos (Kléber, 2000).

De maneira sucinta, durante um ECG é possível observar ondas específicas, que indicam eventos elétricos durante um batimento cardíaco. Estas ondas são identificadas como P, Q, R, S, T e U. A onda P é o primeiro movimento ascendente do ECG e representa a contração atrial, e o bombeamento de sangue para os ventrículos (Smith et al., 2018).

O complexo QRS representa despolarização e contração ventricular. O intervalo PR indica o tempo de propagação do sinal elétrico da região NSA para os ventrículos. E a onda T repolarização ventricular. A onda U é a representação final da repolarização ventricular (Wang et al., 2017).

Uma das primeiras manifestações da isquemia miocárdica é a alteração onda T no segmento ST. O segmento ST representa o intervalo entre despolarização e repolarização dos ventrículos (Kléber, 2000).

O IAM é classificado como Síndrome Coronariana Aguda, e se apresenta de duas formas distintas de acordo com a abrangência isquêmica da parede muscular, e respectiva alterações eletrocardiográficas: Síndrome Coronariana Aguda com elevação do segmento "ST" (correspondente à porção do eletrocardiograma entre o fim da despolarização e o início da repolarização dos ventrículos), e Síndrome Coronariana Aguda Sem elevação do segmento "ST" (Avezum Junior et al., 2015).

Durante a oclusão total de uma artéria coronariana ocorre a alterações nos tecidos musculares epicárdicos, que quando representados eletrocardiográficamente desenvolvem o supradesnivelamento do segmento ST em duas ou mais derivações subsequentes. Esta modificação se inicia já nos primeiros minutos de isquemia miocárdica e pode se estabilizar nas primeiras 12 horas ou voltar à linha de base em até 72 horas. Deve-se considerar a elevação do segmento ST a elevação do ponto J em duas derivações contínuas ou mais, devendo ser medida a partir de uma linha reta traçada da borda superior do segmento PR até a borda superior do segmento ST no nível do ponto J (Denmark et al., 2019).

Assim, o eletrocardiograma quando executado no local de atendimento e interpretado por um profissional habilitado é capaz de reduzir em $34 \%$ o tempo de início de tratamento efetivo, além de proporcionar maiores taxas de tempo ideal para realização de angioplastia quando indicado ocasionando uma redução na mortalidade intra-hospitalar em pacientes com IAM (Avezum Junior et al., 2015).

\section{Diagnóstico de Infarto Agudo do Miocárdio}

Novos conceitos surgiram em revisão publicada em 2018 pela ESC (Europeu Society Cardiology) na quarta definição universal de IAM com a diferenciação entre lesão miocárdica e IAM (Ibanez et al., 2018).

A revisão citada acima determinou que a denominação lesão miocárdica deve ser utilizada quando houver evidência de valores elevados de biomarcadores (troponina) com pelo menos um valor acima do limite máximo de referência (LMR) do percentil 99 (Ibanez et al., 2018). A lesão miocárdica é considerada aguda se houver aumento e/ou queda nos valores da troponina (Denmark et al., 2019).

Além disso, de acordo com Fakhri e colaboradores (2018) há um consenso que o termo IAM deve ser usado quando houver lesão miocárdica com evidência clínica de isquemia miocárdica aguda e com detecção de aumento e/ou queda dos valores do biomarcador com pelo menos um valor acima do (LMR) do percentil 99 associado a um dos critérios a seguir: 
- $\quad$ Sintomas de isquemia miocárdica (IM).

- $\quad$ Alterações eletrocardiográfica evidenciando padrões isquêmicos ou zonas elétricas inativas recentes.

- $\quad$ Alteração no exame de imagem, apresentando áreas isquêmicas com ou sem fibrose, e modificações de contratilidade segmentar do miocárdio.

Identificação de um trombo coronariano por angiografia ou autópsia.

Desta forma, o termo síndrome coronariana aguda, é usado para pacientes em que há suspeita de isquemia miocárdica aguda ou infarto miocárdico, sendo classificadas em três apresentações clínico laboratoriais distintas: angina instável (AI), Infarto Miocárdico (IM) sem supradesnivelamento do segmento ST e IAM com supradesnivelamento do segmento ST (Fakhri et al., 2018).

Os tipos de infarto do miocárdio são classificados de acordo com a provável causa estabelecedora de isquemia miocárdica persistente e são classificados em:

IM TIPO 1: De provável causa aterosclerótica, com ruptura de placa ou erosão perfazendo trombos luminais distais ou não. Pode caracterizar a síndrome coronariana com ou sem supradesnivelamento do segmento ST.

IM TIPO 2: Provável desbalanço entre consumo e oferta de oxigênio. É observado em condições clínicas desfavoráveis em pacientes com doença arterial coronariana estável. Um exemplo imaginável seria um paciente já com redução luminal por doença coronariana aterosclerótica com anemia aguda, ou espasmo coronariano .

IM TIPO 3: Infarto relacionado a morte súbita, de origem supostamente coronariana, com apresentação clínica condizente, entretanto sem provas eletrocardiográficas ou elevação de biomarcadores. Geralmente o diagnóstico deste tipo de IM se dá em autopsias.

IM TIPO 4: Está relacionado com procedimentos hemodinâmicos.

IM TIPO 5: Está relacionado a cirurgia de Revascularização miocárdica em pré, pós e transoperatória (Denmark et al., 2019).

\section{Recomendações quanto ao tratamento do IAM}

Lesões isquêmicas e hipóxicas são os tipos mais comuns de lesões celulares na medicina clínica e são objeto de inúmeras investigações em seres humanos, modelos experimentais de animais e sistemas de cultura de células (Semenza, 2014).

Durante uma lesão isquêmica, ao contrário da hipóxia, a entrega do substrato ao tecido cessa e o metabolismo glicolítico é limitado (Seki \& Fishbein, 2014). No entanto, durante a hipóxia, a produção de energia glicolítica pode prosseguir. Nos tecidos isquêmicos, a geração de energia anaeróbica cessará depois que os substratos glicolíticos se esgotarem ou o processo metabólico forem inibidos pelo acúmulo de metabólitos que não são removidos pela perfusão de órgãos. Portanto, durante a isquemia, a lesão tecidual se desenvolve mais rapidamente do que durante a hipóxia (Eltzschig \& Eckle, 2011).

Durante a isquemia miocárdica, poderá ocorrer o comprometimento de componentes estruturais e bioquímicos vitais, como resultado será observado uma lesão irreversível progressiva que resultará subsequentemente em morte celular (Semenza, 2014).

Se a progressão da lesão celular for interrompida poderá ocorrer o retorno dos substratos metabólico e oxigênio, em um processo denominado reperfusão. No entanto, se o intervalo isquêmico for prolongado, haverá comprometimento celular irreversível e a restauração da perfusão é incapaz de restaurar as condições celulares ideais. Com perda da função mitocondrial, aumento da permeabilidade a moléculas extracelulares e alterações estruturais na membrana plasmática ((Eltzschig \& Eckle, 2011). 
A mudança acelerada no status do tecido na região de risco é evidenciada quase que imediatamente no eletrocardiograma, como uma diminuição na amplitude da onda $\mathrm{R}$ e no aparecimento de uma onda $\mathrm{Q}$, além da perda de enzimas citosólicas e evidência morfológica de morte celular no exame post-mortem. Uma situação paradoxal, portanto, se desenvolve, em que a reoxigenação ou refluxo, embora essencial para a sobrevivência do tecido, pode de fato ser prejudicial (Whelan et al., 2010).

A restauração da irrigação em uma região isquêmica poderá ocasionar danos devidos à restauração do fluxo sanguíneo, denominados lesões de reperfusão. Contraditoriamente, o retorno sanguíneo causa lesão tecidual adicional, denominada lesão por reperfusão (Dotta et al., 2018)

Os mecanismos moleculares e celulares decorrentes da lesão de reperfusão são diversos e incluem estresse oxidativo, alteração da membrana mitocondrial, dano mitocondrial, sobrecarga de cálcio, hipercontração, apoptose, resposta inflamatória, ativação plaquetária e disfunção endotelial. Que poderão ocasionar as seguintes manifestações clínicas: lesão por reperfusão letal, disfunção microvascular, atordoamento miocárdico e arritmias por reperfusão (van der Pol et al., 2019).

Desta forma, é necessário que medidas iniciais sejam tomadas a fim de conter as lesões decorrentes tanto da isquemia quanto da reperfusão, portanto, há mais de um século, a suplementação de oxigênio tem sido utilizada rotineiramente no tratamento de pacientes com suspeita de IAM principalmente nas horas iniciais ao mesmo, sendo realizado $3 \mathrm{~L} / \mathrm{min}$ a $100 \%$, através de cateter nasal oxigênio em pacientes com saturação menor que 90\% (Avezum Junior et al., 2015). Mas ressalta-se que a eficácia da oxigenoterapia de rotina em pacientes com IAM permanece altamente incerta.

\section{Analgesia}

Devido à morte celular do miocárdio ou isquemia, o IAM geralmente é acompanhado de dor no peito, aperto ou desconforto que pode irradiar para ombros, braços, costas, pescoço ou mandíbula (Moore et al., 2019).

O alívio dos sintomas derivados do IAM não somente é importante para o bem-estar do paciente, mas também porque o estresse ocasionado pelo processo doloroso induz um aumento maciço da liberação de catecolaminas pelo sistema nervoso simpático, levando a efeitos circulatórios sistêmicos como aumento da pressão arterial, frequência cardíaca e volume sistólico (Abdi \& Basgut, 2016). Como resultado, estas alterações podem interferir negativamente no suprimento metabólico do miocárdio e resultar em uma extensão aumentada do infarto piorando o quadro do paciente.

Desde 1923, quando James MacKenzie sugeriu o uso de morfina para o tratamento de pacientes cardíacos, este opióide ainda permanece como sendo o agente analgésico de escolha no IAM (Abdi \& Basgut, 2016). Além do grande poder analgésico, a morfina promove sedação leve com diminuição da ansiedade, e por consequência, diminuição da liberação de catecolaminas com redução do consumo do oxigênio e demanda metabólica, mas a utilização de benzodiazepínicos para pacientes com ansiedade persistente também é recomendado (Mussi et al., 2014)..

\section{Anti-agregantes plaquetários}

Uma das primeiras estratégias medicamentosas a ser realizada é a administração de ácido acetilsalicílico (AAS) assim que se observa a dor típica observada no IAM que atuará na inibição da produção de TXA2 através da inibição irreversível da enzima cicloxigenase 1 (COX-1) (Southerland, 2016).

A casca do salgueiro tem sido utilizada como medicamento há mais de 3.500 anos. Sumérios e egípcios se beneficiavam dos poderes analgésicos desta planta, mas foi somente em 1828 que os cristais amarelos da casca foram extraídos por Johann Buchner, e o ingrediente ativo denominado de salicilina (Desborough \& Keeling, 2017).

Em 1853, Charles Frederic Gerhardt criou o ácido acetilsalicílico pela primeira vez, mas ele não usou nem comercializou essa versão modificada do ácido salicílico (Khan et al., 2016). Quando em 1897, Felix Hoffman, um químico 
alemão que trabalhava para a empresa Bayer, também modificou o ácido salicílico para criar o ácido acetilsalicílico (AAS) ou aspirina, que resultou na sua potente disseminação (Wick, 2012).

No início dos anos 50, antes dos mecanismos de ação da aspirina terem sido elucidados, Lawrence Craven relatou sua experiência com a utilização da aspirina. Onde observou que a prescrição da mesma ocasionava complicações hemorrágicas graves em pacientes submetidos a amigdalectomias (Craven, 1950). Desta forma, Craven deduziu que o fármaco poderia prevenir eventos trombóticos, passando a recomendar aspirina a seus pacientes. Seus estudos sobre os efeitos antitrombóticos como agente anti-plaquetário só foram resgatados uma década depois (Miner \& Hoffhines, 2007).

Como descrito anteriormente, o AAS tem um efeito antiagregante devido o bloqueio irreversível da COX-1 derivada do ácido araquidônico. O ácido araquidônico é responsável pela síntese do tromboxano A2 (TxA2), e a utilização de AAS ocasiona consequentemente a inibição da TxA2 e ambos com ações antiagregantes (Miner \& Hoffhines, 2007).

O uso precoce de AAS reduz o risco de morte em 23\% (isolado) e $43 \%$ quando associado ao fibrinolítico, além de reduzir o risco de re-oclusão coronariana e novos eventos isquêmicos (Avezum Junior et al., 2015). A prescrição é de 160-325 mg diariamente, exceto para pacientes com alergia ao medicamento ou com hemorragia no trato digestório.

Assim, observa-se que apesar de a aspirina ao ter mais de 100 anos desde sua ampla distribuição, a mesma continua a ocupar um lugar de destaque no tratamento de doenças cardiovasculares.

Sabendo que as plaquetas desempenham um papel fundamental na fisiopatologia do IAM, inibidores da ativação plaquetária como o 5'-difosfato de adenosina (ADP), um dos mediadores mais importantes da ativação plaquetária, foram estudados como alvos medicamentosos (Koupenova et al., 2017).

Dos receptores ADP acoplados à proteína $\mathrm{G}$, o receptor P2Y12, de distribuição restrita, sendo seletivamente expresso em megacariócitos, plaquetas e algumas regiões cerebrais, desempenha um papel central na ativação plaquetária bem como no crescimento e estabilização de um trombo (Gachet \& Hechler, 2013).

Sendo assim, devido à seletividade dos receptores P2Y12, medicamentos denominados tienopiridinas, que atuam com especificidade sobre este receptor foram desenvolvidos. Entre eles pode-se citar as tienopiridinas como o clopidogrel, ticlopidina e prasugrel antagonistas irreversíveis, bem como, o ticagrelor e o cangrelor, antagonistas reversíveis. Cada um desses medicamentos tem eficácia comprovada em grandes ensaios clínicos, e podem ser usados de acordo com suas propriedades e indicações específicas (Abdi \& Basgut, 2016; Gachet \& Hechler, 2013).

O clopidogrel, considerado um dos antagonistas mais utilizados, deverá ser prescrito inicialmente na forma de dose de ataque, podendo variar entre 300-600 mg dependendo da idade do paciente, seguido da dose de manutenção $75 \mathrm{mg} / \mathrm{dia}$. Mas é importante que se frise que esta medicação apresenta duas grandes limitações, a primeira, um início de ação lenta, uma vez que este é um pró-farmaco que necessita ser metabolizado pelas enzimas hepáticas, para que se obtenha o metabólito ativo, a segunda, a falta de resposta adequada em alguns pacientes (Khan et al., 2016).

Respeitando os grandes estudos e indicações em associações do clopidogrel com o AAS, esta associação verificada obteve reduções significativas de mortalidade e morbidade nos eventos isquêmicos cardiovasculares e cerebrovasculares. (Somuncu et al., 2019).

\section{Nitratos}

Os nitratos, juntamente com terapias antiplaquetárias e analgésicas adicionais, formam a base do tratamento inicial do IAM (van der Pol et al., 2019).

O óxido nítrico (NO) é um mediador gasoso de curta duração com um papel fundamental no sistema cardiovascular, e com propriedades importantes durante os danos da isquemia e reperfusão. Assim, alterações na via de sinalização do NO estão correlacionadas com o aumento da morbidade do IAM (Parati et al., 2016). 
Os nitratos orgânicos, como trinitrato de glicerila (também conhecido como nitroglicerina) é utilizados na medicina cardiovascular, atuam como um gerador de NO, mas são limitados devido desenvolvimento de tolerância e disfunção endotelial (Graboys \& Lown, 2003).

Os nitratos são catalisados por sulfidrilas à NO na túnica média, levando a um aumento na produção de monofosfato cíclico de guanosina, reduzindo o cálcio intracelular, causando o relaxamento muscular e vasodilatação. Consequentemente, ocorre a redução na força de contração e demanda de oxigênio (Ferreira \& Mochly-Rosen, 2012).

O tratamento medicamentoso com nitroglicerina é de $0,4 \mathrm{mg}$, já o mononitrato de isossorbida apresenta dosagem de $5,0 \mathrm{mg}$; e o dinitrato de isossorbida, $5,0 \mathrm{mg}$, sendo administrados no máximo três doses, com intervalos de 5 minutos entre as mesmas (Piegas et al., 2015).

\section{Reperfusão coronariana}

Quando se gerencia a escolha do método de tentativa na reperfusão coronariana nos pacientes com IAM, é necessário atentar para as condições técnicas e estruturais encontradas no hospital referência em que o paciente é atendido (Antman, 2008). Em situação ideal, de suporte técnico e estrutural, com sala de hemodinâmica e hemodinamicista 24 horas, com tempo de chegada do paciente ao hospital até o procedimento hemodinâmico (tempo porta-balão) menor do que 90 minutos, com experiência de muitos procedimentos realizados, discute-se na literatura a superioridade da terapia de reperfusão mecânica ou angioplastia (Ibanez et al., 2018).

\section{Intervenção Coronariana Percutânea}

A intervenção coronariana percutânea (ICP) por balão foi à primeira técnica percutânea utilizada para reperfusão no IAM, por isso o nome utilizado para descrever o espaço temporal mais importante para o tratamento, o tempo porta-balão, que é definido pelo tempo entre a chegada do paciente no hospital e a abertura ou liberação da luz coronariana, ao inflar o balão na região afetada (Park et al., 2019).

Novas técnicas percutâneas foram empregadas com o passar do tempo, entre estas citam-se os stents coronarianos, farmacológicos e não farmacológicos, os dispositivos de trombectomia e tromboaspiração (Olivera Escalona, 2015).

Atualmente a ICP primária é a terapia de escolha, desde que respeitado o tempo de até 90 minutos da admissão do paciente e que seja realizado em um centro com pelo menos 200 procedimentos ao ano, por operadores que realizem ao menos 75 procedimentos/ano (Fonseca \& Izar., 2016).

A ICP primária mostrou-se superior à terapia trombolítica, pois é capaz de restaurar o fluxo coronariano e o desempenho hemodinâmico em mais de $90 \%$ dos casos, assim como, reduzir as taxas de IAM e suas sequelas, aumentando a sobrevida (Ibanez et al., 2018).

\section{Terapia de Reperfusão Química}

A terapia fibrinolítica foi validada por ensaios de grande magnitude. estes trabalhos demonstraram a preservação da função ventricular, redução da mortalidade e do tamanho do infarto. A metanálise FTT (Fibrinolytc Therapy Trials), incluindo todos os grandes estudos testando fibrinolíticos, demonstraram que a medida em que aumentava o tempo para o início do emprego do fibrinolítico após o início dos sintomas do IAM, menor era sua eficiência, não havendo qualquer benefício após 12 horas (Avezum Junior et al., 2015).

Desta forma, a terapia fibrinolítica é recomendada dentro de 12 horas do início dos sintomas e quanto mais o paciente posterga a procura médica, menores são as chances de reperfusão da coronária alvo, sendo estipulado que o tempo máximo 
correlacionado às melhores chances de eficácia terapêutica são de até 3 horas do início dos sintomas (Baruzzi et al., 2018; Dotta et al., 2018).

Atualmente no Brasil é possível encontrar três diferentes agentes fibrinolíticos específicos, com propriedade distintas, que requerem orientação e protocolos para serem utilizados que seguem abaixo:

a) A estreptoquinase (SK) é uma proteína bacteriana altamente purificada extraída do filtrado de cultura de estreptococos beta-hemolíticos do grupo $\mathrm{C}$ de Lancefield. Ressalta-se que alguns pacientes podem ter resistência ao tratamento com estreptoquinase devido a possível resistência imunoinduzida por eventuais processos infecciosos com as bactérias superfícies-sensíveis. Além disso, durante a sua infusão não é incomum variações pressóricas e de frequência cardíaca, gerando instabilidade hemodinâmica ao paciente com necessidade de intervenções adjuntas frequentes (Baruzzi et al., 2018).

b) A alteplase (tPA), formada por um grupamento de proteínas formando uma enzima que se liga à fibrina com maior afinidade do que a SK, convertendo a plasminogênio em plasmina na superfície da molécula de fibrina. Atualmente um dos fibrinolíticos mais utilizados nos hospitais. Tem como fator negativo ao seu emprego, uma meia vida muito curta e requer a administração de uma heparina para não haver reoclusão da coronária obstruída (Baruzzi et al., 2018).

c) A tenecteplase (TNK), trombolítico que possui maior eficácia no tratamento quando relacionada a alteplase por ter meia vida de duração maior e especificidade maior à fibrina. Além do mais, tem-se a vantagem de poder ser administrada em dose única, em bolus, sendo opção fantástica ao tratamento do IAM ainda em fase pré hospitalar. O que impossibilita sua implantação nas rotina do sistema único de saúde é o seu elevado custo (Fonseca \& Izar., 2016).

\section{Conclusão}

O IAM é a principal causa de morte no mundo, com diminuição significativa da qualidade de vida entre os sobreviventes. A principal etipatogenia é a ruptura de uma lesão aterosclerótica levando a formaçao de um trombo que diminui o fluxo de sangue resultando em uma isquemia miocardial persistente ou transitória, com sintomas clinicos como a dor no peito e/ou falta de ar, dor epigástrica e dor no braço.

Seu diagnóstico, bem como tratamentos, devem ser os mais precoces possíveis. Em 1971, Eugene Braunwald propôs a hipótese que a severidade e a extensão da isquêmica poderiam ser alteradas através da intervenção adequada em até 3 horas após a oclusão coronariana (Kjekshus, 1974). Desde então, houve uma melhora substancial no diagnóstico e tratamentos do IAM. Mas é importante destacar que o tempo desde o início dos sintomas (oclusão da artéria coronária) até a instituição do tratamento (reperfusão química ou mecânica) é diretamente proporcional à ocorrência de eventos clinicamente relevantes, podendo determinar o sucesso da reperfusão coronariana e por seguinte, a diminuição da morbidade e mortalidade do paciente. Assim, ressalta-se que os benefícios do tratamento é tempo-dependente.

Conforme anteriormente discutido, a maioria das mortes por IAM ocorrem nas primeiras horas de manifestação da doença, principalmente em razão de arritmias ventriculares. O prognóstico e as implicações entre o tempo porta-balão e o tempo entre o início dos sintomas até a chegada do paciente ao hospital com terapias efetivas de reperfusão miocárdica, apresentam benefício claro na redução de mortalidade e morbidade dos pacientes com tempos menores do que 3 horas.

No contexto de regionalização demográfica e recursos locais, cada estado e até mesmo regiões do estado, estão organizados muitas vezes de maneira não eficiente para roteirizar e homogeneizar o atendimento dos pacientes com dor torácica caracterizada como síndrome coronariana aguda (Oliveira et al., 2018).

Assim, quando o sistema de saúde apresenta um direcionamento com ações de preventivas e de implementações para uma patologia específica, aumenta-se o número de diagnóstico, reduz-se o atraso no atendimento e consequentemente, a mortalidade também diminui. 
Mas é essencial para que o IAM apresente uma redução na morbimortalidade, a instrução sobre os sinais e sintomas, bem como, a necessidade de intervenção precoce. Principalmente em se tratando das mulheres, que apresentam sintomas atípicos e tendem a não reconhecer as manifestações do IAM.

Sabendo que constantemente acontecem avanços nas diferentes áreas do conhecimento, sugere-se que futuramente as técnicas e intervenções descritas neste artigo, sejam revisitadas e se necessário, reescritas, para o correto acompanhamento científico.

\section{Agradecimentos}

Ao UNIEDU, programa do Estado de Santa Catarina, executado pela Secretaria de Estado da Educação (SED).

\section{Referências}

Abdi, A., \& Basgut, B. (2016). An evidence-based review of pain management in acute myocardial infarction. Journal of Cardiology \& Clinical Research, 4(4), 1067. https://doi.org/10.1038/ijo.2008.36

Abreu, L. M. (2019). Time is muscle. Arquivos Brasileiros de Cardiologia, 112(4), 408-409. https://doi.org/10.5935/abc.20190059

Antman, E. M. (2008). Time Is Muscle. Translation Into Practice. Journal of the American College of Cardiology, 52(15), 1216-1221. https://doi.org/10.1016/j.jacc.2008.07.011

Avezum Junior, Á., Feldman, A., Carvalho, A. C. de C., Sousa, A. C. S., Mansur, A. de P., Bozza, A. E. Z., Falcão, B. de A. A., Markman Filho, B. M., Polanczyk, C. A., Gun, C., Serrano Junior, C. V., Oliveira, C. C. de, Moreira, D., Précoma, D. B., Magnoni, D., Albuquerque, D. C. de, Romano, E. R., Stefanini, E., \& Santos, E. S. Dos. (2015). V Diretriz da Sociedade Brasileira de Cardiologia sobre Tratamento do Infarto Agudo do Miocárdio com Supradesnível do Segmento ST. Arquivos Brasileiros de Cardiologia, 105(2 Suppl 1), 1-121. https://doi.org/10.5935/abc.20150107

Baruzzi, A. C. do, Stefanini, E., \& Manzo, G. (2018). Fibrinolíticos: indicações e tratamento das complicações hemorrágicas TT - Fibrinolytics: indications and treatment of hemorrhagic complications. Revista da Sociedade de Cardiologia do Estado de Säo Paulo, 28(4), 421-427.

Braunwald, E. (1998). Evolution of the management of acute myocardial infarction: A 20th century saga. Lancet, 352(9142), 1771-1774. https://doi.org/10.1016/S0140-6736(98)03212-7

Cowley, R. A. (1975). A total emergency medical system for the State of Maryland. Maryland Medical Journal, 24(7), 37-45.

Craven, L. L. (1950). Acetylsalicylic acid, possible preventive of coronary thrombosis. Annals of Western Medicine and Surgery, 4(2), 95.

Dalen, J. E., Alpert, J. S., Goldberg, R. J., \& Weinstein, R. S. (2014). The epidemic of the 20th century: Coronary heart disease. American Journal of Medicine, 127(9), 807-812. https://doi.org/10.1016/j.amjmed.2014.04.015

Denmark, K. T., Bax, J. J., Morrow, D. A., Task, A., Members, F., Kristian, C., Denmark, T., White, H. D., Zealand, N., Denmark, H. M., Uk, B., Germany, H. A. K., Germany, C. W. H., Januzzi, J. L., Angeles, M., Garcia, A., Uk, S. R. U., Canty, J. M., Lyon, A. R., Uk, K. A. A. F. (2019). Fourth universal definition of myocardial infarction (2018). 237-269. https://doi.org/10.1093/eurheartj/ehy462

Desborough, M. J. R., \& Keeling, D. M. (2017). The aspirin story - from willow to wonder drug. British Journal of Haematology, 177(5), 674-683. https://doi.org/10.1111/bjh.14520

Dotta, G., Fonseca, F. A. H., Izar, M. C. de O., Souza, M. T. de, Moreira, F. T., Pinheiro, L. F. M., Barbosa, A. H. P., Caixeta, A. M., Póvoa, R. M. S., Carvalho, A. C., \& Bianco, H. T. (2018). Regional QT Interval Dispersion as an Early Predictor of Reperfusion in Patients with Acute Myocardial Infarction after Fibrinolytic Therapy. Arquivos Brasileiros de Cardiologia, 112, 20-29. https://doi.org/10.5935/abc.20180239

Eltzschig, H. K., \& Eckle, T. (2011). Ischemia and reperfusion-from mechanism to translation. In Nature Medicine. 1391-1401. https://doi.org/10.1038/nm.2507

Fakhri, Y., Melgaard, J., Andersson, H. B., Schoos, M. M., Birnbaum, Y., Graff, C., Sejersten, M., Kastrup, J., \& Clemmensen, P. (2018). Automatic electrocardiographic algorithm for assessing severity of ischemia in ST-segment elevation myocardial infarction. International Journal of Cardiology, 268, 18-22. https://doi.org/10.1016/j.ijcard.2018.04.057

Ferreira, J. C. B., \& Mochly-Rosen, D. (2012). Nitroglycerin use in myocardial infarction patients: Risks and benefits. Circulation Journal : Official Journal of the Japanese Circulation Society, 76(1), 15-21. http://www.ncbi.nlm.nih.gov/pubmed/22040938\%0Ahttp://www.pubmedcentral.n ih.gov/articlerender.fcgi?artid=PMC3527093

Fonseca, \& Izar. (2016). Diagnóstico Das Síndromes Coronarianas Agudas E Modelo Sistematizado De Atendimento Em Unidades De Dor Torácica. Soc. Cardiol. Estado de São Paulo (Rev. Da SOSESP), 26(2), 72-85.

Gachet, C., \& Hechler, B. (2013). The P2Y receptors and thrombosis. Wiley Interdisciplinary Reviews: Membrane Transport and Signaling, 2(6), 241-253. https://doi.org/10.1002/wmts.97

Graboys, T., \& Lown, B. (2003). Nitroglycerin: The “Mini” Wonder Drug. Circulation, 108(11), e78-9. https://doi.org/10.1161/01.CIR.0000086629.67552.3A 
Ibanez, B., James, S., Agewall, S., Antunes, M. J., Bucciarelli-Ducci, C., Bueno, H., Caforio, A. L. P., Crea, F., Goudevenos, J. A., Halvorsen, S., Hindricks, G., Kastrati, A., Lenzen, M. J., Prescott, E., Roffi, M., Valgimigli, M., Varenhorst, C., Vranckx, P., Widimský, P., Gale, C. P. (2018). 2017 ESC Guidelines for the management of acute myocardial infarction in patients presenting with ST-segment elevation. European Heart Journal, 39(2), 119-177. https://doi.org/10.1093/eurheartj/ehx393

Kaptoge, S., Pennells, L., De Bacquer, D., Cooney, M. T., Kavousi, M., Stevens, G., Riley, L. M., Savin, S., Khan, T., Altay, S., Amouyel, P., Assmann, G., Bell, S., Ben-Shlomo, Y., Berkman, L., Beulens, J. W., Björkelund, C., Blaha, M., Blazer, D. G., Di Angelantonio, E. (2019). World Health Organization cardiovascular disease risk charts: revised models to estimate risk in 21 global regions. The Lancet Global Health, 7(10), e1332-e1345. https://doi.org/10.1016/S2214-109X(19)30318-3

Khan, J. N., Greenwood, J. P., Nazir, S. A., Lai, F. Y., Dalby, M., Curzen, N., Hetherington, S., Kelly, D. J., Blackman, D., Peebles, C., Wong, J., Flather, M., Swanton, H., Gershlick, A. H., \& McCann, G. P. (2016). Infarct size following treatment with second-versus third-generation P2Y12 antagonists in patients with multivessel coronary disease at ST-segment elevation myocardial infarction in the CvLPRIT study. Journal of the American Heart Association, 31(6), e003403. https://doi.org/10.1161/JAHA.116.003403

Kjekshus, J. K. (1974). Factors influencing infarct size following coronary artery occlusion. In J.Oslo City Hosp. (Vol. 24, Issues 11-12, pp. 155-175). http://www.ncbi.nlm.nih.gov/pubmed/5540853

Kléber, A. G. (2000). ST-segment elevation in the electrocardiogram: A sign of myocardial ischemia. Cardiovascular Research, 45(1), 111-118. https://doi.org/10.1016/S0008-6363(99)00301-6

Koupenova, M., Kehrel, B. E., Corkrey, H. A., \& Freedman, J. E. (2017). Thrombosis and platelets: An update. European Heart Journal, 38(11), 785-791. https://doi.org/10.1093/eurheartj/ehw550

Mahmooda, S. S., Levy, D., Vasan, R. S., \& Wang, T. J. (2014). The Framingham Heart Study and the epidemiology of cardiovascular diseases: A historical perspective. Lancet, 383(9921), 1933-1945. https://doi.org/10.1016/S0140-6736(13)61752-3.The

Mann, D., Zipes, D., Libby, P., \& Bonow, R. (2014). Braunwald's Heart Disease: A Textbook of Cardiovascular Medicine. Cleveland Clinic Journal of Medicine, 51(3), 565-565. https://doi.org/10.3949/ccjm.51.3.565-a

Miner, J., \& Hoffhines, A. (2007). The discovery of aspirin’s antithrombotic effects. Texas Heart Institute Journal, 34(2), 179-186.

Moore, A., Goerne, H., Rajiah, P., Tanabe, Y., Saboo, S., \& Abbara, S. (2019). Acute Myocardial Infarct. Radiologic Clinics of North America, 57(1), 45-55. https://doi.org/10.1016/j.rcl.2018.08.006

Mozaffarian, D., Benjamin, E. J., Go, A. S., Arnett, D. K., Blaha, M. J., Cushman, M., Das, S. R., De Ferranti, S., Després, J. P., Fullerton, H. J., Howard, V. J., Huffman, M. D., Isasi, C. R., Jiménez, M. C., Judd, S. E., Kissela, B. M., Lichtman, J. H., Lisabeth, L. D., Liu, S., Turner, M. B. (2016). Executive summary: Heart disease and stroke statistics-2016 update: A Report from the American Heart Association. Circulation, 133(4), 447-454. https://doi.org/10.1161/CIR.0000000000000366

Mussi, F. C., Mendes, A. S., Queiroz, T. L. de, Costa, A. L. S., Pereira, Á., \& Caramelli, B. (2014). Pre-hospital delay in acute myocardial infarction: judgement of symptoms and resistance to pain. Revista Da Associacão Medica Brasileira (1992), 60(1), 63-69. http://www.ncbi.nlm.nih.gov/pubmed/24918855

Oliveira, J. C., Costa, L., Oliveira, S., Oliveira, J. C., Carvalho, I. D. De, Almeida-santos, M. A., Clair, T., Munareto, R., Maia, L. A., Flávio, L., Prado, A., Silveira, F. S., Nascimento, T. A., Pereira, E. J., Barreto, R. V., Moraes, E. V., Mendonça, J. T. De, Carlos, A., Pós-graduação, N. De, Brasil, S. E. (2018). Disparidades no Tratamento do Infarto Agudo do Miocárdio entre Usuários dos Sistemas de Saúde Público e Privado em Sergipe. International Journal of Cardiovascular Sciences, 31(4), 339-358.

Olivera Escalona, Á. L. (2015). Reperfusión coronaria en el infarto agudo del miocardio con elevación del segmento ST: importancia del contexto y tiempo de aplicación. Medisan, 19(10), 1259-1267.

Parati, G., Lombardi, C., Castagna, F., Mattaliano, P., Filardi, P. P., Agostoni, P., \& Italian Society of Cardiology (SIC) Working Group on Heart Failure members. (2016). Heart failure and sleep disorders. Nature Reviews. Cardiology, 13(7), 389-403. https://doi.org/10.1038/nrcardio.2016.71

Park, J., Choi, K. H., Lee, J. M., Kim, H. K., Hwang, D., Rhee, T., Kim, J., Park, T. K., Yang, J. H., Song, Y. Bin, Choi, J., Hahn, J., Choi, S., Koo, B., Chae, S. C., Cho, M. C., Kim, C. J., Kim, J. H., Jeong, M. H., Kim, H. (2019). Prognostic Implications of Door-to-Balloon Time and Onset-to-Door Time on Mortality in Patients With ST-Segment-Elevation Myocardial Infarction Treated With Primary Percutaneous Coronary Intervention. Journal of the American Heart Association, 8(9), e012188. https://doi.org/10.1161/jaha.119.012188

Piegas, L., Timerman, A., Feitosa, G., Nicolau, J., Mattos, L., Andrade, M., Avezum, A., Feldman, A., De Carvalho, A., Sousa, A., Mansur, A., Bozza, A., Falcão, B., Markman Filho, B., Polanczyk, C., Gun, C., Serrano Junior, C., Oliveira, C., Moreira, D., Mathias Junior, W. (2015). V Diretriz da Sociedade Brasileira de Cardiologia Sobre Tratamento do Infarto Agudo do Miocárdio com Supradesnível do Segmento ST. Arquivos Brasileiros de Cardiologia, 105(2), 1-121. https://doi.org/10.5935/abc.20150107

Ribeiro, J. L. P (2014). Revisão de Investigação e Evidência Científica. Psicologia, Saúde \& Doenças, 15(3), 671-682.

Reimer, K. A., Lowe, J. E., Rasmussen, M. M., \& Jennings, R. B. (1977). The wavefront phenomenon of ischemic cell death. 1. Myocardial infarct size vs duration of coronary occlusion in dogs. Circulation, 56(5), 786-794. https://doi.org/10.1161/01.CIR.56.5.786

Seki, A., \& Fishbein, M. C. (2014). Ischemic Heart Disease. In Pathobiology of Human Disease: A Dynamic Encyclopedia of Disease Mechanisms. https://doi.org/10.1016/B978-0-12-386456-7.03305-0

Semenza, G. L. (2014). Oxygen Sensing, Hypoxia-Inducible Factors, and Disease Pathophysiology. Annual Review of Pathology: Mechanisms of Disease, 9 , 47-71. https://doi.org/10.1146/annurev-pathol-012513-104720

Smith, V., Arunthavanathan, S., Nair, A., Ansermet, D., Da Silva Costa, F., \& Wallace, E. M. (2018). A systematic review of cardiac time intervals utilising 
Research, Society and Development, v. 11, n. 3, e23811326447, 2022

(CC BY 4.0) | ISSN 2525-3409 | DOI: http://dx.doi.org/10.33448/rsd-v11i3.26447

non-invasive fetal electrocardiogram in normal fetuses. BMC Pregnancy and Childbirth, 18(1), 370. https://doi.org/10.1186/s12884-018-2006-8

Somuncu, M. U., Demir, A. R., Karakurt, S. T., Karakurt, H., \& Karabag, T. (2019). Long term cardiovascular outcome based on aspirin and clopidogrel responsiveness status in young ST-elevated myocardial infarction patients. Arquivos Brasileiros de Cardiologia, 112(2) 138-146. https://doi.org/10.5935/abc.20180251

Southerland, A. (2016). The History of Aspirin: from Willow Bark to Thomas Edison in the 20th Century. Neurology, 86(16), 2.391.

van der Pol, A., van Gilst, W. H., Voors, A. A., \& van der Meer, P. (2019). Treating oxidative stress in heart failure: past, present and future. In European Journal of Heart Failure. 21(4), 425-435. https://doi.org/10.1002/ejhf.1320

Vigmond, E. J., \& Stuyvers, B. D. (2016). Modeling our understanding of the His-Purkinje system. Progress in Biophysics and Molecular Biology, 120(1-3), 179-188. https://doi.org/10.1016/j.pbiomolbio.2015.12.013

Wang, G., Liu, R., Chang, Q., Xu, Z., Zhang, Y., \& Pan, D. (2017). Electrocardiogram: His bundle potentials can be recorded noninvasively beat by beat on surface electrocardiogram. BMC Cardiovascular Disorders, 17(1), 1-8. https://doi.org/10.1186/s12872-017-0516-3

Whelan, R. S., Kaplinskiy, V., \& Kitsis, R. N. (2010). Cell Death in the Pathogenesis of Heart Disease: Mechanisms and Significance. Annual Review of Physiology, 72, 19-44. https://doi.org/10.1146/annurev.physiol.010908.163111

WHO | Cardiovascular diseases (CVDs). (2019). WHO. https://www.who.int/cardiovascular_diseases/en/

Wick, J. Y. (2012). Aspirin: A history, a love story. Consultant Pharmacist, 27(5), 322-329. https://doi.org/10.4140/TCP.n.2012.322 